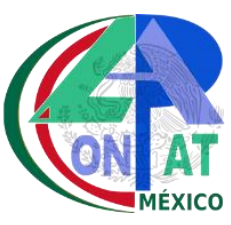

\title{
EVALUACIÓN DEL ESTADO DE CONDICIÓN FÍSICO DE VIVIENDAS EN ZONAS DE RIESGO DE LA CIUDAD DE TUXTLA GUTIÉRREZ, CHIAPAS, MÉXICO
}

\author{
F. ALONSO ${ }^{1}$, J. CASTELLANOS ${ }^{1}$, M. NAZAR ${ }^{1}$, A. ALONSO ${ }^{2}$, D. PIMENTEL ${ }^{2}$, C. GORDILLO ${ }^{2}$ \\ ${ }^{1}$ PTC Facultad de Ingeniería, Universidad Autónoma de Chiapas, México \\ ${ }^{2}$ Estudiantes de la Facultad de Ingeniería, Universidad Autónoma de Chiapas, México
}

\begin{abstract}
RESUMEN
Se presenta el desarrollo de un modelo para la evaluación de estructuras que permite determinar el estado de condición de una vivienda. Este modelo de evaluación propone el análisis de la estructura desde el punto de vista funcional y estructural, aplicando para cada uno de ellos factores de peso dependiendo del deterioro que tenga la estructura al momento de inspeccionarla. Con estos dos factores se establecen parámetros e índices que califican a la estructura en alguno de los cinco estados de condición propuestos para el modelo.
\end{abstract}

Palabras clave: Evaluación, índice funcional, estructural, deterioro. 


\section{INTRODUCCIÓN}

En investigaciones anteriores se han desarrollado campañas de inspección a viviendas de interés social en zonas de riesgo en la ciudad de Tuxtla Gutiérrez, Chiapas, México, (Alonso, 2007; Gómez, 2008; Pimentel, 2018) con la finalidad de determinar el estado de condición y poder evaluar el daño que tienen dependiendo de la patología presentada. Se han utilizado diversos modelos para la evaluación, pero no han cumplido con los resultados esperados, por lo que se propuso desarrollar un modelo en base a modelos experimentados con adecuaciones acordes a las condiciones de deterioro que se presentan en esas zonas. Puede mencionarse que la importancia de este modelo radica en que evalúa a la estructura desde el punto de vista funcional y estructural. El índice funcional permite evaluar a la estructura desde el punto de vista del servicio que ésta presta al usuario, permitiendo realizar debidamente las funciones para la cual fue construida dicha estructura. El índice estructural proporciona el deterioro estructural que presenta la estructura al momento de la inspección, y se calcula tomando en cuenta el deterioro que puede tener en losas, muros, elementos de confinamiento o refuerzo y cimentación.

\section{ANTECEDENTES}

La ciudad de Tuxtla Gutiérrez es la capital del Estado de Chiapas, mismo que se ubica en el sureste de México, siendo así una de las ciudades más extensas, pobladas y urbanizadas del Estado, aunque no tenga tanta importancia turística ni cultural, como otras ciudades, es el centro económico y político de Chiapas. Se localiza en la región central del Estado, a $16^{\circ} 45^{\prime} 11^{\prime}$ ' Latitud Norte y $93^{\circ} 06^{\prime}$ '56' Longitud Oeste y $550 \mathrm{msnm}$. Tiene una extensión territorial de $412.4 \mathrm{~km}^{2}$.

En los últimos años, la ciudad de Tuxtla Gutiérrez ha tenido una derrama económica bastante importante generada por la iniciativa privada, que han invertido en la construcción y desarrollo de complejos hoteleros y comerciales, así también esta derrama se ha dirigido a la construcción de unidades habitacionales lo que ha producido un incremento en la infraestructura urbana y en la población.

Desafortunadamente, la ciudad de Tuxtla Gutiérrez se encuentra ubicada en la Depresión Central presentando un relieve montañoso tanto al sur como al norte, por lo que varias unidades habitacionales de interés social se están construyendo en zonas que se pueden denominar de riesgo, debido a la inestabilidad del suelo donde se desplantan. Aunado a esto, cada unidad cuenta con diferentes características estructurales por lo que no se puede normalizar un proceso constructivo para cada una de ellas ni homogenizar el tipo de estructuración (sistema de piso, sistema estructural y sistema de cimentación), debido a esto frecuentemente se presentan en dichas estructuras diversas patologías estructurales que en muchas ocasiones no es factible identificar la causa que lo produce y por lo tanto no se les aplica la acción correctiva que requiere el problema presentado.

Otro factor que se puede considerar como una causa de fallo, es que el subsuelo de la ciudad de Tuxtla Gutiérrez presenta algunas características especiales (arcillas expansivas) que hacen que las propuestas de cimentación deben ser bien estudiadas para cada caso que se requiera, ya que dependiendo de la zona donde se va construir, se deberán realizar propuestas de mejoramiento o estabilización del suelo y de la cimentación en que se deberá desplantar la estructura para evitar que se presentes fallos durante el proceso de cambios volumétricos del terreno.

Por último, la ciudad de Tuxtla Gutiérrez se encuentra comprendida en la zona C (Figura 1) dentro de la Regionalización Sísmica de la Comisión Federal de Electricidad (CFE, 2008), lo que provoca junto con el acelerado ritmo de construcción que se presenta en las unidades habitacionales, los diversos procesos constructivos y en algunos casos la mala calidad en los materiales o en la supervisión, que se presenten una serie de patologías estructurales provocadas por la acción dinámica y que pueden poner en riesgo la estabilidad estructural de una vivienda. 


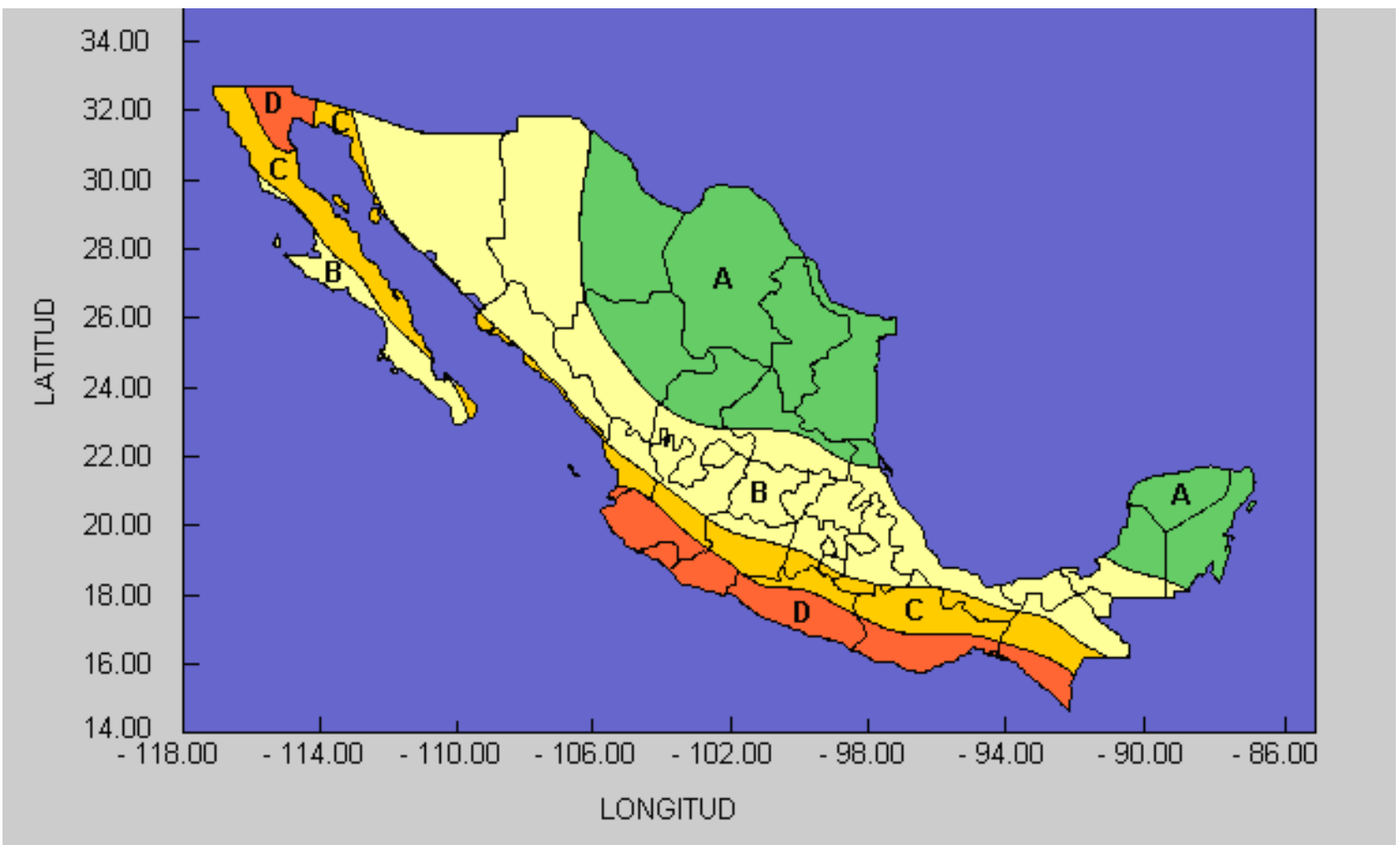

Figura .1 Regionalización Sísmica de la República Mexicana (CFE, 2008)

\section{MODELO DE EVALUACIÓN DEL ESTADO DE CONDICIÓN}

En esta investigación se presenta un modelo para evaluar el estado de condición físico de una estructura basándose en los datos recolectados en campañas de inspección. Se toma como base el modelo propuesto en Alonso (2007), aunque con dos variantes principales, las cuales se describen a continuación.

Este modelo de evaluación propone el análisis de la estructura desde el punto de vista funcional y estructural, aplicando para cada uno de ellos factores de peso dependiendo del deterioro que tenga la estructura al momento de inspeccionarla.

Por lo tanto, el estado de condición de una estructura es evaluada a partir del Índice de Estado de Condición (IEC)

$$
I E C=I F+I E
$$

Donde $\quad$ IF $=$ Índice Funcional

$\mathrm{IE}=$ Índice Estructural

A partir de este índice, se calcula el estado de condición de la estructura inspeccionada tomando en cuenta los valores establecidos en la Tabla 1, donde se describe el estado de condición dependiendo del IEC obtenido, su descripción y la magnitud de daño según las NTC-17. 
Tabla 1. Estado de Condición de una Estructura

\begin{tabular}{|c|c|c|c|}
\hline IEC & $\begin{array}{l}\text { Estado de } \\
\text { Condición }\end{array}$ & Descripción & $\begin{array}{l}\text { Magnitud de daño } \\
\text { (NTC-17) }\end{array}$ \\
\hline 0.00 & 1 - Excelente & $\begin{array}{l}\text { La estructura no presenta } \\
\text { ningún daño }\end{array}$ & \\
\hline $0.01-4.99$ & 2 - Bueno & $\begin{array}{l}\text { La estructura presenta } \\
\text { daños menores que se } \\
\text { pueden solucionar con } \\
\text { mantenimientos mínimos }\end{array}$ & $\begin{array}{l}\text { Insignificante, que no afecta de manera } \\
\text { relevante la capacidad estructural } \\
\text { (resistente y de deformación). La } \\
\text { reparación será de tipo superficial. }\end{array}$ \\
\hline $5.00-9.99$ & 3 - Aceptable & \begin{tabular}{|lrr} 
La & estructura presenta \\
daños & menores & de \\
urgencia & de reparación a \\
largo & plazo & y \\
mantenimientos menores
\end{tabular} & $\begin{array}{l}\text { Ligero, cuando afecta ligeramente la } \\
\text { capacidad estructural. Se requieren } \\
\text { medidas de reparación sencillas para la } \\
\text { mayor parte de elementos y de modos } \\
\text { de comportamiento. }\end{array}$ \\
\hline $10.00-14.99$ & 4 - Regular & $\begin{array}{l}\text { La estructura presenta } \\
\text { daños mayores que } \\
\text { pueden poner en peligro la } \\
\text { estabilidad de la estructura } \\
\text { y requiere acciones de } \\
\text { mantenimiento a mediano } \\
\text { plazo }\end{array}$ & $\begin{array}{l}\text { Moderado, cuando afecta } \\
\text { medianamente la capacidad estructural. } \\
\text { La rehabilitación de los elementos } \\
\text { dañados depende del tipo de elemento } \\
\text { y modo de comportamiento. }\end{array}$ \\
\hline $15.00-19.99$ & 5 - Malo & $\begin{array}{l}\text { La estructura presenta } \\
\text { daños mayores } r \text { que } \\
\text { necesitan acciones de } \\
\text { reforzamiento a corto } \\
\text { plazo }\end{array}$ & $\begin{array}{l}\text { Severo, cuando el daño afecta } \\
\text { significativamente la capacidad } \\
\text { estructural. La rehabilitación implica } \\
\text { una intervención amplia, con } \\
\text { reemplazo o refuerzo de algunos } \\
\text { elementos. }\end{array}$ \\
\hline $20.00-59.00$ & 6 - Dañado & $\begin{array}{l}\text { La estructura presenta } \\
\text { daños mayores con } \\
\text { acciones de reforzamiento } \\
\text { inmediatas }\end{array}$ & $\begin{array}{l}\text { Muy grave, cuando el daño ha } \\
\text { deteriorado a la estructura al punto que } \\
\text { su desempeño no es confiable. Abarca } \\
\text { el colapso total o parcial. La } \\
\text { rehabilitación involucra el reemplazo o } \\
\text { refuerzo de la mayoría de los } \\
\text { elementos, o incluso la demolición total } \\
\text { o parcial. }\end{array}$ \\
\hline
\end{tabular}

\section{Análisis del Índice Funcional (IF)}

El modelo anterior no hacía referencia al estado de servicio de la estructura desde el punto de vista del usuario, por lo tanto, en este modelo se propone el uso de un índice funcional que permite evaluar a la estructura desde el punto de vista del servicio que ésta presta al usuario, así como realizar debidamente las funciones para la cual fue construida dicha estructura.

Este índice se calcula con la siguiente ecuación (2):

$$
I F=F F * F M
$$

Donde $\quad \mathrm{FF}=$ Factor Funcional

$\mathrm{FM}=$ Factor de Mantenimiento 
El Factor Funcional (FF) es un factor que permite evaluar la estructura desde el punto de vista funcional y se le asignan los valores establecidos en la Tabla 2, dependiendo de la apreciación del inspector al momento de la evaluación.

Tabla 2. Valores para el Factor Funcional

\begin{tabular}{|c|l|}
\hline FF & \multicolumn{1}{|c|}{ Descripción } \\
\hline 0 & La estructura está en perfecto estado \\
\hline 1 & Presencia de suciedad en muros, losas y pisos \\
\hline 2 & $\begin{array}{l}\text { Presencia de pintura descamada, puertas y ventanas con defectos } \\
\text { menores, desprendimiento de recubrimiento }\end{array}$ \\
\hline 3 & $\begin{array}{l}\text { Pequeñas manchas de humedad con filtraciones mínimas, desperfectos } \\
\text { eléctricos e hidráulicos, falta de azulejos y losetas, cristales rotos }\end{array}$ \\
\hline 4 & $\begin{array}{l}\text { Desprendimientos en muros, losas y pisos, filtraciones mayores, } \\
\text { puertas y ventanas en mal estado, faltan cristales }\end{array}$ \\
\hline 5 & Filtraciones excesivas, puertas y ventanas dañadas \\
\hline 6 & La estructura es inhabitable \\
\hline
\end{tabular}

Los valores propuestos para cada observación de esta tabla se tomaron del estudio realizado a 77 viviendas inspeccionadas en la ciudad de Tuxtla Gutiérrez ubicadas en zonas de riesgo (Figura 2), agrupando las observaciones más recurrentes que provocaban un fallo en el uso de la vivienda.

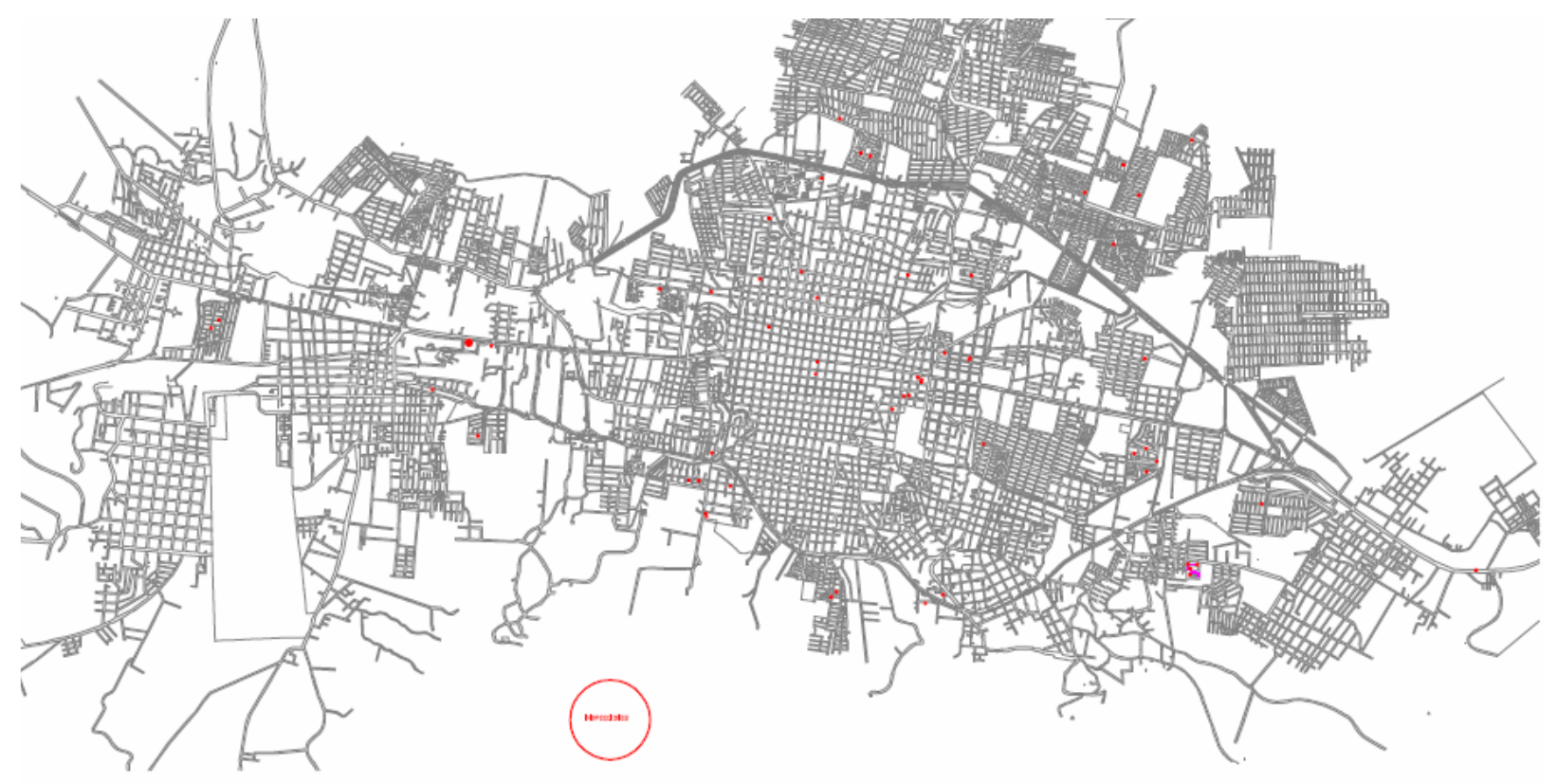

Figura 2. Ubicación de viviendas inspeccionadas

El Factor de Mantenimiento (FM) es un valor que se asigna dependiendo del tipo de mantenimiento que requiere la estructura para que proporcione el mejor estado de servicio con respecto a los requerimientos funcionales del usuario. Existen dos tipos de mantenimientos: preventivo y correctivo. La mayoría de los investigadores definen al mantenimiento preventivo como aquella actividad relacionada con la limpieza, pintura o reposición de recubrimientos de elementos estructurales, mientras que el mantenimiento correctivo abarca una gran gama de actividades que van desde la rehabilitación, reparación, reforzamiento o reposición de elementos estructurales para que puedan soportar las acciones a las que están sujetos. Para el caso de establecer los valores que intervienen en el factor de 
mantenimiento, se tomaron en cuenta solo las actividades que se realizan en el mantenimiento preventivo, clasificándolo en menor, medio y mayor. Los valores que se le asignan se describen en la Tabla 3.

Tabla 3. Valores para el Factor de Mantenimiento

\begin{tabular}{|c|l|}
\hline FM & \multicolumn{1}{|c|}{ Descripción } \\
\hline 0 & La estructura no requiere de ningún mantenimiento \\
\hline 1 & Mantenimiento menor, limpieza general de la estructura \\
\hline 2 & $\begin{array}{l}\text { Mantenimiento medio, resanes, reposición de cristales, azulejos, } \\
\text { losetas, reparación menor hidráulica y eléctrica, pintura general, } \\
\text { aplicación de sellador en losa }\end{array}$ \\
\hline 3 & $\begin{array}{l}\text { Mantenimiento mayor, reparación de recubrimientos, ventanas, } \\
\text { puertas, impermeabilizante general }\end{array}$ \\
\hline
\end{tabular}

Los valores anteriores se tomaron de las campañas de inspección realizadas a viviendas en la ciudad de Tuxtla Gutiérrez y tomando como base que las viviendas y cada uno de los elementos que las componen se encuentran sometidas a un uso permanente lo que provoca un deterioro normal de cada uno de los componentes y para que la vivienda se mantenga en buen estado de servicio a través de su vida útil se debe usar adecuadamente. Lo anterior implica tener cuidado de su aseo y de conocer la función de cada elemento que forma la vivienda.

Los beneficios que se generan del buen uso y de un mantenimiento adecuado en la vivienda son los siguientes:

- Evitar el deterioro de la vivienda, prolongando el buen estado de ella a lo largo de su vida útil.

- Mejorar la apariencia física de la misma.

- Prevenir los daños en elementos estructurales

- Identificar problemas menores y darle solución oportuna,

\section{Análisis del Índice Estructural (IE)}

El índice estructural proporciona el deterioro estructural que presenta la estructura al momento de la inspección, y se calculó tomando en cuenta el deterioro que puede tener la vivienda en los cuatro componentes fundamentales de su sistema estructural que son: losas, muros, elementos de confinamiento o refuerzo y cimentación.

La expresión que calcula el índice estructural (IE) es la siguiente:

$$
I E=D L+D M+D E C+D C
$$

Donde $\quad$ DL $=$ Deterioro en Losa

$\mathrm{DM}=$ Deterioro en Muros

$\mathrm{DEC}=$ Deterioro en Elementos de Confinamiento

$\mathrm{DC}=$ Deterioro en Cimentación

Cada uno de estos deterioros proporciona un índice del daño que puede tener el elemento estructural en base a tres factores que son: el Factor de Daño, el Factor de Acción y el Factor de Urgencia.

El Factor de Daño (FD) representa un índice que es establecido por el ingeniero encargado de la evaluación y depende del daño observado en el elemento estructural al momento de la inspección y de 
la probable causa que lo origina.

El Factor de Acción (FA) representa el nivel de mantenimiento correctivo que se requiere para que, al menos, el elemento estructural regrese a su nivel de desempeño de diseño. Este tipo de mantenimiento puede ir desde reparaciones menores hasta reforzamientos considerables en los elementos que componen la vivienda.

Y por último, el Factor de Urgencia (FU) indica la urgencia de la intervención o acción que requiere el elemento tomando en cuenta el daño que se observa al momento de la inspección.

Los valores para cada uno de los factores que determinan el deterioro en cada elemento estructural fueron establecidos de acuerdo a las observaciones patológicas obtenidas en las campañas de inspección realizadas en diversas zonas de la ciudad de Tuxtla Gutiérrez, tomando ciertas características estructurales y de uso al momento de realizar el muestreo aleatorio de las viviendas a inspeccionar.

En figura 3 se muestran las patologías más frecuentes que se observaron durante las campañas de inspección, clasificadas con respecto a los cuatro componentes fundamentales en que se divide su sistema estructural.

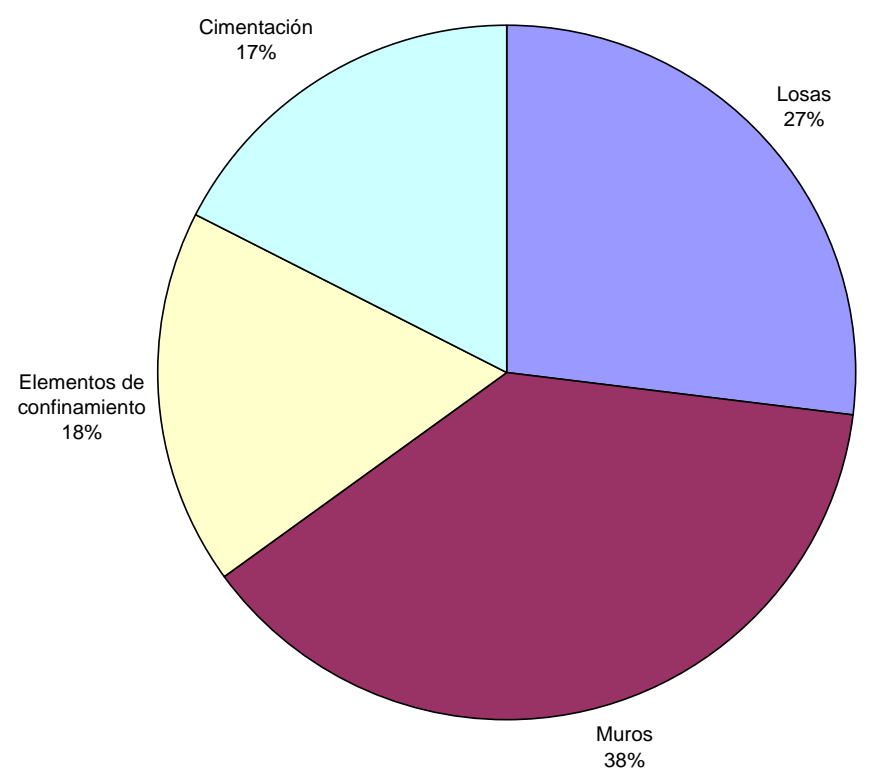

Figura 3. Porcentaje de deterioro en cada elemento estructural

Con base al análisis realizado a las observaciones de las patologías obtenidas durante las campañas de inspección y a las propuestas realizadas por autores como Bellmunt et al. (2000), Emmons (2005), Escolá (1993), García (2002) y Gómez (2008), se propusieron los valores para cada uno de los factores que determinan el deterioro en los elementos estructurales propuestos.

Deterioro Losas (DL)

El DL se calcula entonces con la siguiente expresión

$$
D L=F D_{L} * F A_{L} * F U_{L}
$$

El índice para el Factor de Daño en el elemento losa $\left(F D_{L}\right)$ se estableció, como se mencionó anteriormente, principalmente del estudio realizado a las viviendas inspeccionadas y se determinó de acuerdo a las patologías observadas y lo que representaba cada una de ellos con respecto al daño total en el elemento. La asignación de este valor depende de la apreciación del evaluador. Se proponen valores de referencia de estos índices en la Tabla 6. 
Tabla 6.- Factores propuestos dependiendo de las patologías analizadas para $\mathrm{FD}_{\mathrm{L}}$

\begin{tabular}{|c|c|}
\hline FD $_{\mathrm{L}}$ & Descripción \\
\hline 0 & - No se presenta ninguna patología \\
\hline $0.01-0.05$ & $\begin{array}{l}\text { - Pequeñas fisuras menores a } 10 \mathrm{~cm} \text { de longitud } \\
\text { - Descamado de pintura provocado por humedad. } \\
\text { - Manchas de suciedad }\end{array}$ \\
\hline $0.05-0.10$ & $\begin{array}{l}\text { - Fisuras entre } 10 \text { y } 20 \mathrm{cms} \text { de longitud } \\
\text { - Descamado de recubrimiento en menos del } 10 \% \text { del área }\end{array}$ \\
\hline $0.10-0.25$ & $\begin{array}{l}\text { - Bloques de fisuras entre } 5 \text { y } 50 \mathrm{cms} \text { por flexión o contracción. } \\
\text { - Descamados de recubrimiento del } 10-20 \% \\
\text { - Pequeñas manchas provocadas por Humedad } \\
\text { - Pequeñas filtraciones con goteo en la losa }\end{array}$ \\
\hline $0.25-0.50$ & $\begin{array}{l}\text { - Grietas menores a } 5 \mathrm{~mm} \text { de espesor con longitudes menores a } 10 \mathrm{cms} \\
\text { - Vibración notable de la losa } \\
\text { - Descamados de recubrimiento del } 30 \text { - } 50 \% \\
\text { - Manchas de humedad con presencia de óxido o eflorescencia } \\
\text { - Desprendimiento de concreto en menos del } 10 \%\end{array}$ \\
\hline $0.50-0.75$ & $\begin{array}{l}\text { - Grietas de más de } 5 \mathrm{~mm} \text { de espesor con longitud variable } \\
\text { - Manchas de humedad con desprendimiento del recubrimiento y presencia de } \\
\text { óxido o excesiva eflorescencia } \\
\text { - Desprendimiento del concreto con acero de refuerzo visible en un } 20 \% \\
\text { - Vibraciones excesivas }\end{array}$ \\
\hline $0.75-1.00$ & $\begin{array}{l}\text { - Filtraciones excesivas con manchas de óxido y desprendimiento del concreto } \\
\text { - Grietas de más de } 5 \mathrm{~mm} \text { de espesor con longitud variable y con manchas de } \\
\text { oxidación } \\
\text { - Acero de refuerzo visible y corroído } \\
\text { - Desprendimientos de recubrimiento con acero expuesto en más de } 20 \%\end{array}$ \\
\hline
\end{tabular}

Para determinar el Factor de Daño, en el caso de las losas, se observó que la mayoría de las patologías que se presentaron en las viviendas analizadas, requerían de reforzamiento total, por lo que se establecieron estos valores como se indica en la Tabla 7.

Tabla 7. Valores para el Factor de Acción en Losas

\begin{tabular}{|c|l|}
\hline $\mathbf{F A}_{\mathbf{L}}$ & \multicolumn{1}{|c|}{ Descripción } \\
\hline 0 & No requiere ninguna reparación \\
\hline 1 & Reparaciones menores que consisten en limpieza de pequeños escamados, sello de fisuras \\
\hline 2 & Reparaciones menores que consisten en resane de grietas o pequeños desprendimientos \\
\hline 3 & $\begin{array}{l}\text { Reparaciones mayores que consisten en reparación de desprendimientos y limpieza o } \\
\text { reposición de acero corroído }\end{array}$ \\
\hline 4 & Reparaciones mayores que consisten en reforzamiento de la losa \\
\hline
\end{tabular}

En la Tabla 8 se presentan las acciones requeridas en las losas para que estas recuperen al menos su nivel de desempeño original. 
Tabla 8. Valores para el Factor de Urgencia en Losas

\begin{tabular}{|c|c|}
\hline $\mathbf{F U}_{\mathbf{L}}$ & Descripción \\
\hline 1 & Acción a largo plazo \\
\hline 2 & Acción a mediano plazo \\
\hline 3 & Acción a corto plazo \\
\hline
\end{tabular}

\section{Deterioro Muros (DM)}

El DM se calcula entonces con la siguiente expresión

$$
D M=F D_{M} * F A_{M} * F U_{M}
$$

El Factor de Daño en muros ( $\left.\mathrm{FD}_{\mathrm{M}}\right)$ es un factor que va de 0 a 1 y depende de la apreciación del evaluador y consiste en un factor de la cantidad de daño que tiene el elemento Muro de la estructura en general, en la Tabla 9 se proponen valores de referencia para el $\mathrm{FD}_{\mathrm{M}}$.

Tabla 9.- Factores propuestos dependiendo de las patologías analizadas para $\mathrm{FD}_{\mathrm{M}}$

\begin{tabular}{|c|c|}
\hline FDM $_{M}$ & Descripción \\
\hline 0 & - No se presenta ninguna patología \\
\hline $0.01-0.05$ & $\begin{array}{l}\text { - Pequeñas fisuras por contracción en el recubrimiento. } \\
\text { - Descamado de pintura. }\end{array}$ \\
\hline $0.05-0.10$ & $\begin{array}{l}\text { - Pequeñas fisuras menores de } 50 \mathrm{~cm} \text { de longitud en el muro. } \\
\text { - Desprendimiento de recubrimiento hasta en } 10 \% \text { de la superficie del muro. }\end{array}$ \\
\hline $0.10-0.20$ & $\begin{array}{l}\text { - Fisuras entre } 50 \text { y } 100 \mathrm{~cm} \text { de longitud en el muro. } \\
\text { - Desprendimiento de recubrimiento hasta en } 10 \% \text { de la superficie del muro. }\end{array}$ \\
\hline $0.20-0.30$ & $\begin{array}{l}\text { - Fisuras entre } 100 \text { y } 150 \mathrm{~cm} \text { de longitud } \\
\text { - Desprendimiento de recubrimiento entre } 10 \text { y } 20 \% \text { de la superficie del muro. }\end{array}$ \\
\hline $0.30-0.50$ & $\begin{array}{l}\text { - Grietas hasta de } 50 \mathrm{~cm} \text { de longitud en el muro. } \\
\text { - Desprendimiento de recubrimiento más del } 20 \% \text { de la superficie del muro. }\end{array}$ \\
\hline $0.50-0.60$ & $\begin{array}{l}\text { - Grietas entre } 50 \text { y } 150 \mathrm{~cm} \text { de longitud en el muro. } \\
\text { - Desprendimiento de recubrimiento más del } 20 \% \text { de la superficie del muro. }\end{array}$ \\
\hline $0.80-1.00$ & - Grietas mayores de $150 \mathrm{~cm}$ de longitud en el muro. \\
\hline
\end{tabular}

Cabe mencionar, que el evaluador puede hacer uso de su experiencia y calificar con un índice que el considere el adecuado, aunque no esté especificado así en la tabla anterior.

En las Tablas 10 y 11 se presentan los valores para $\mathrm{FA}_{\mathrm{M}}$ y $\mathrm{FU}_{\mathrm{M}}$, respectivamente.

Tabla 10. Valores para el Factor de Acción en Muros

\begin{tabular}{|c|l|}
\hline $\mathbf{F A}_{\mathbf{M}}$ & \multicolumn{1}{|c|}{ Descripción } \\
\hline 0 & No requiere ninguna reparación \\
\hline 1 & Reparaciones menores que consisten en limpieza de pequeños escamados, sello de fisuras \\
\hline 2 & Reparaciones menores que consisten en resane de grietas o pequeños desprendimientos \\
\hline 3 & Reparaciones mayores que consisten en reparación de parte del muro dañado \\
\hline 4 & Reparaciones mayores que consisten en reforzamiento o cambio del muro \\
\hline
\end{tabular}


Tabla 11. Valores para el Factor de Urgencia en Muros

\begin{tabular}{|c|c|}
\hline FUM $_{\mathbf{M}}$ & Descripción \\
\hline 1 & Acción a largo plazo \\
\hline 2 & Acción a mediano plazo \\
\hline 3 & Acción a corto plazo \\
\hline
\end{tabular}

\section{Deterioro en Elementos de Confinamiento (DEC)}

El DEC se calcula entonces con la siguiente expresión

$$
D E C=F A_{E C} * F U_{E C} * F D_{E C}
$$

El FDEC es un factor que va de 0 a 1 y depende de la apreciación del evaluador y consiste en un factor de la cantidad de daño que tiene los Elementos de Confinamiento de la estructura en general, en la Tabla 12 se proponen algunos valores para el FD $\mathrm{EC}_{\text {. }}$

Tabla 12.- Factores propuestos dependiendo de las patologías analizadas para FD EC

\begin{tabular}{|c|c|}
\hline FDEC & Descripción \\
\hline 0 & - No se presenta ninguna patología \\
\hline $0.01--0.05$ & $\begin{array}{l}\text { - Pequeñas fisuras por contracción en el recubrimiento } \\
\text { - Descamado de pintura }\end{array}$ \\
\hline $0.05-0.10$ & - Pequeñas fisuras menores de $5 \mathrm{~cm}$ de longitud en el elemento de confinamiento. \\
\hline $0.10-0.20$ & $\begin{array}{l}\text { - Fisuras entre } 5 \text { y } 10 \mathrm{~cm} \text { de longitud en el elemento de confinamiento. } \\
\text { - Pequeñas apariciones de corrosión o eflorescencia }\end{array}$ \\
\hline $0.20-0.40$ & $\begin{array}{l}\text { - Grietas hasta de } 10 \mathrm{~cm} \text { de longitud en el confinamiento. } \\
\text { - Manchas de corrosión o eflorescencia hasta de un } 10 \% \text { de la superficie del } \\
\text { confinamiento }\end{array}$ \\
\hline $0.40-0.50$ & $\begin{array}{l}\text { - Grietas de entre } 10 \text { y } 15 \mathrm{~cm} \text { de longitud en el confinamiento. } \\
\text { - Manchas de corrosión o eflorescencia entre un } 10 \text { y } 30 \% \text { de la superficie del } \\
\text { confinamiento } \\
\text { - Desprendimiento del concreto con acero de refuerzo visible en un } 10 \% \text { de la } \\
\text { superficie del confinamiento. }\end{array}$ \\
\hline $0.50-0.70$ & $\begin{array}{l}\text { - Grietas de entre } 15 \text { y } 20 \mathrm{~cm} \text { de longitud en el confinamiento. } \\
\text { - Manchas de corrosión o eflorescencia mayores del } 30 \% \text { de la superficie del } \\
\text { confinamiento } \\
\text { - Desprendimiento del concreto con acero de refuerzo corroído visible entre un } \\
10 \text { y } 40 \% \text { de la superficie del confinamiento. }\end{array}$ \\
\hline $0.70-1.0$ & $\begin{array}{l}\text { - Grietas con longitudes mayores a } 20 \mathrm{~cm} \text { en el confinamiento. } \\
\text { - Desprendimiento del concreto con acero de refuerzo corroído visible en más } \\
\text { del } 40 \% \text { de la superficie del confinamiento. }\end{array}$ \\
\hline
\end{tabular}

Al considerarse con más importancia estructural el deterioro en este tipo de elementos, se determinó un factor de acción de mayor importancia que en los elementos anteriores. En la Tabla 13 se presentan los valores para $F A_{E C}$. 
Tabla 13. Valores para el Factor de Acción en Elementos de Confinamiento

\begin{tabular}{|c|l|}
\hline FAEc & \multicolumn{1}{|c|}{ Descripción } \\
\hline 0 & No requiere ninguna reparación \\
\hline 1 & Reparaciones menores que consisten en limpieza de pequeños escamados, sello de fisuras \\
\hline 2 & Reparaciones menores que consisten en resane de grietas o pequeños desprendimientos \\
\hline 3 & Reparaciones mayores que consisten en reparación de parte del confinamiento dañado \\
\hline 4 & Reparaciones mayores que consisten en reforzamiento o cambio del confinamiento \\
\hline 5 & $\begin{array}{l}\text { Reparaciones mayores que consisten en reforzamiento total del confinamiento usando } \\
\text { elementos nuevo y apuntalamientos }\end{array}$ \\
\hline
\end{tabular}

Sin embargo los valores para las acciones de urgencia de la reparación son las mismas que los elementos anteriores al tomar la consideración de que no son fallos de peligro inminente si no son reforzados, el deterioro puede ser controlado con el apuntalamiento. En la Tabla 14 se presentan los valores para FAEC y FUEC

Tabla 14. Valores para el Factor de Urgencia en Elementos de Confinamiento

\begin{tabular}{|c|c|}
\hline FUEC & Descripción \\
\hline 1 & Acción a largo plazo \\
\hline 2 & Acción a mediano plazo \\
\hline 3 & Acción a corto plazo \\
\hline
\end{tabular}

\section{Deterioro Cimentación (DC)}

El DC se calcula entonces con la siguiente expresión

$$
D C=F A_{C} * F U_{C} * F D_{C}
$$

La determinación de los índices para el deterioro en la cimentación no pudo ser realizado tomando en cuenta las observaciones de las patologías en las campañas de inspección, debido a que muchas de las patologías que se pusieron como causa problemas de cimentación fueron realizadas de manera subjetiva ya que las campañas de inspección fueron solo de carácter visual, sin embargo con el apoyo de la literatura revisada se determinaron estos índices, los cuales se indican en la Tabla 15.

Tabla 15.- Factores propuestos dependiendo de las patologías analizadas para $\mathrm{FD}_{\mathrm{C}}$

\begin{tabular}{|c|c|}
\hline Porcentaje & Descripción \\
\hline 0 & - No se presenta ninguna patología \\
\hline $0.01-0.05$ & - Pequeñas fisuras de no más de $50 \mathrm{~cm}$ de longitud en la cimentación. \\
\hline $0.05-0.20$ & $\begin{array}{l}\text { - Fisuras entre } 50 \text { y } 100 \mathrm{~cm} \text { de longitud en la cimentación. } \\
\text { - Desprendimiento del concreto en menos del 5\% de la superficie. }\end{array}$ \\
\hline $0.20-0.40$ & $\begin{array}{l}\text { - Fisuras entre } 100 \text { y } 150 \mathrm{~cm} \text { de longitud en la cimentación. } \\
\text { - Desprendimiento del concreto } 5 \text { y el } 10 \% \text { de la superficie. } \\
\text { - Manchas de corrosión o eflorescencia hasta de un } 10 \% \text { de la superficie }\end{array}$ \\
\hline $0.40-0.60$ & $\begin{array}{l}\text { - Grietas menores de } 50 \mathrm{~cm} \text { de longitud en la cimentación. } \\
\text { - Desprendimiento del concreto } 10 \text { y el } 15 \% \text { de la superficie. } \\
\text { - Asentamientos o levantamiento por cambio volumétrico provocando fisuras de } \\
\text { hasta } 5 \mathrm{~cm} \text { al pie de columna o muro de carga. }\end{array}$ \\
\hline
\end{tabular}




\begin{tabular}{|l|l|}
\hline $0.60-0.80$ & - Grietas entre 50 y $100 \mathrm{~cm}$ de longitud en la cimentación. \\
& - Desprendimiento de concreto con acero visible entre el 15 y el $20 \%$ \\
& - Asentamientos o levantamiento por cambio volumétrico provocando fisuras de \\
& hasta $10 \mathrm{~cm}$ de longitud al pie de columna o muro de carga. \\
\hline $0.80-1.00$ & - Grietas de más $100 \mathrm{~cm}$ de longitud en la cimentación. \\
& - Desprendimiento del concreto con acero de refuerzo corroído visible en más del \\
& $20 \%$. \\
& - Asentamientos o levantamiento por cambio volumétrico provocando grietas \\
& mayores a $10 \mathrm{~cm}$ de longitud, provocando mal funcionamiento en puertas y \\
& ventanas.
\end{tabular}

Al igual que el deterioro en elementos de confinamiento, un daño en la cimentación requiere de acciones de aseguren la estabilidad de la estructura, en la Tabla 16 se presentan los valores para FAC.

Tabla 16. Valores para el Factor de Acción en Cimentación

\begin{tabular}{|c|l|}
\hline FAc & \multicolumn{1}{|c|}{ Descripción } \\
\hline 0 & No se requiere ninguna reparación \\
\hline 1 & Reparaciones menores que consisten en limpieza de pequeños escamados, sello de fisuras \\
\hline 2 & Reparaciones menores que consisten en resane de grietas o pequeños desprendimientos \\
\hline 3 & Reparaciones mayores que consisten en reparación de parte de la cimentación \\
\hline 4 & $\begin{array}{l}\text { Reparaciones mayores que consisten en reforzamiento o cambio de los elementos de la } \\
\text { cimentación }\end{array}$ \\
\hline 5 & $\begin{array}{l}\text { Reparaciones mayores que consisten en reforzamiento total de la cimentación usando } \\
\text { elementos nuevo y apuntalamientos }\end{array}$ \\
\hline
\end{tabular}

Y también al ser este elemento estructural el encargado de transmitir las cargas actuantes en la estructura al terreno de desplante, las acciones que se deben de realizar si se presenta un daño que ponga en peligro la estabilidad de la estructura deben ser inmediatas. La Tabla 17 presenta los valores para $\mathrm{FU}_{\mathrm{C}}$.

Tabla 17. Valores para el Factor de Urgencia en Cimentación

\begin{tabular}{|c|l|}
\hline FUC $_{\mathbf{C}}$ & \multicolumn{1}{|c|}{ Descripción } \\
\hline 1 & Acción a largo plazo \\
\hline 2 & Acción a mediano plazo \\
\hline 3 & Acción a corto plazo \\
\hline 4 & Acción Inmediata \\
\hline
\end{tabular}

\section{VALIDACIÓN DEL MODELO}

Para validar el modelo se tomaron las inspecciones realizadas durante la investigación, en distintas zonas de riesgo de la ciudad de Tuxtla Gutiérrez, procurando que la mayoría de ellas se llevaran a cabo en las denominadas zonas de riesgo de la Ciudad.

Cada una de las inspecciones fue capturada en el SIEVP (Alonso \& Chávez, 2009) con la finalidad de poder tener un apoyo informático como soporte al realizar el proceso de evaluación de las viviendas.

Con las observaciones obtenidas para cada vivienda inspeccionada se procedió a evaluar el estado de condición de cada vivienda tomando en cuenta no solo el modelo propuesto sino el que se encuentra 
implementado en el SIEVP.

Considerando que la evaluación fue realizada por el equipo de trabajo que realizó los dos modelos propuestos se propuso que las viviendas inspeccionadas fueran evaluadas por tres grupos distintos de supervisores, explicándoles la forma de evaluación de cada uno de los modelos, en las figuras 4 y 5 se presentan las gráficas de los resultados obtenidos por el equipo de trabajo y los 3 equipos de supervisores.

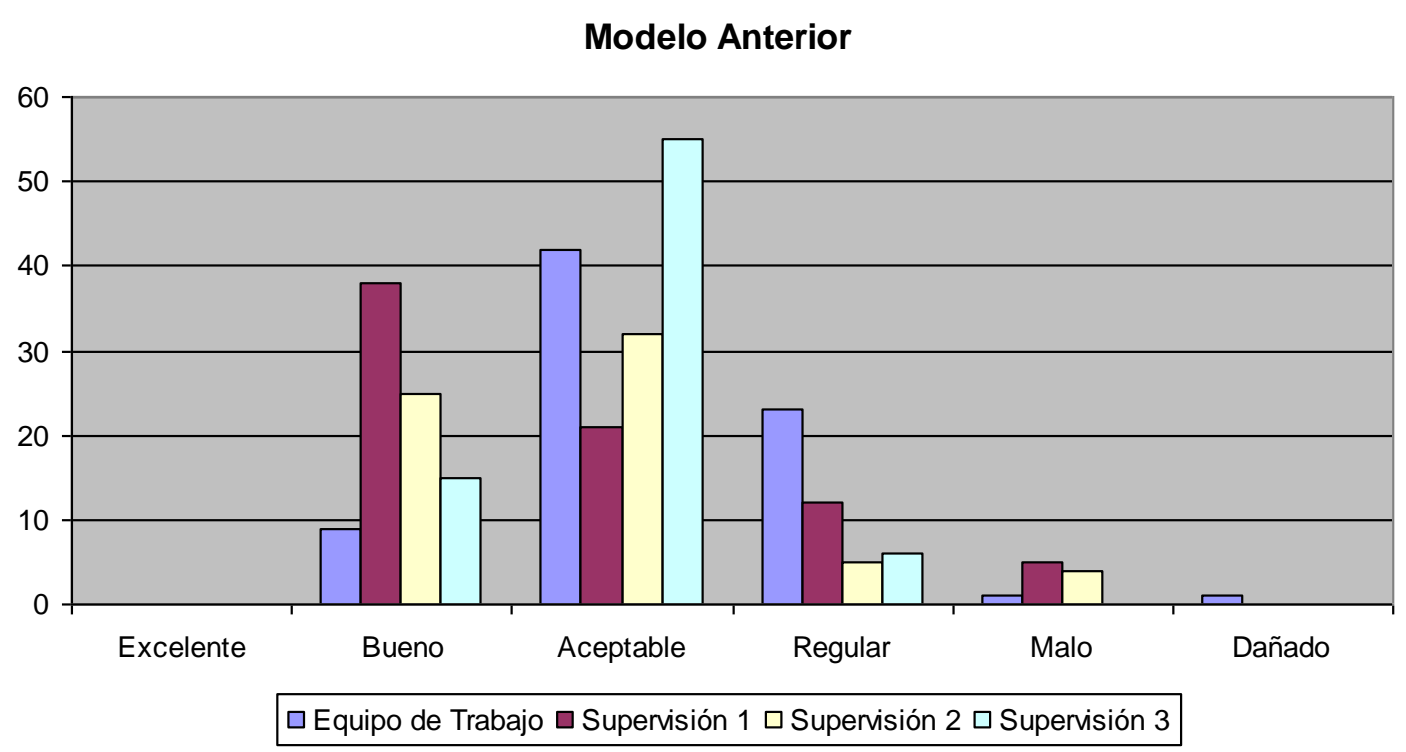

Figura 4. Comparativa de los Estados de Condición de las viviendas evaluadas con el modelo anterior

\section{Modelo Propuesto}

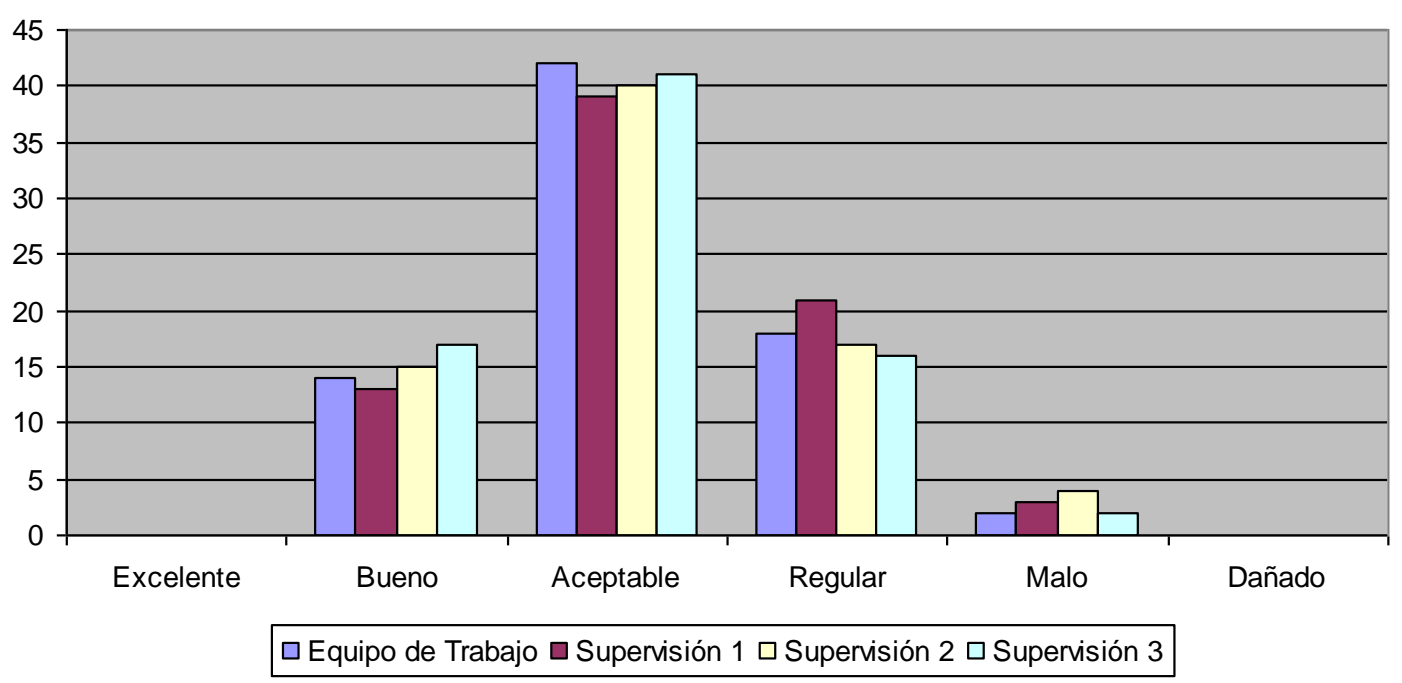

Figura 5. Comparativa de los Estados de Condición de las viviendas evaluadas con el modelo propuesto

Si se observan ambas figuras, se puede apreciar que los resultados obtenidos por el modelo propuesto son en promedio similares, lo que significa que la metodología propuesta en el nuevo modelo propone herramientas más claras para poder evaluar el estado de condición de viviendas de una manera más similar y homogénea 


\section{CONCLUSIONES}

Se evaluaron las 77 viviendas inspeccionadas usando el modelo propuesto y se compararon los estados de condición de cada vivienda con los propuestos con el modelo anterior, obteniéndose como resultado diferencias entre cada modelo.

Estas diferencias se debieron principalmente al que el modelo anterior solo se basa en el dictamen visual del estado de la vivienda desde el punto de vista estructural obtenido de la inspección, en cambio el modelo propuesto, al usar tanto el índice funcional como estructural, proporcionó valores de estados de condición más reales a la observación visual de la vivienda.

Este modelo apoya a la toma de decisiones al usar el Factor de Acción y el de Urgencia de reparación, los cuales son determinados de acuerdo al daño que presenta el elemento analizado.

Sin embargo, es necesario continuar con la validación del modelo para calibrar los rangos que se proponen para cada uno de los índices.

\section{REFERENCIAS}

Alonso, F. (2007). “Inspección y evaluación de los edificios de concreto del H. Ayuntamiento Municipal de Tuxtla Gutiérrez” Unidades de Vinculación Docente, Universidad Autónoma de Chiapas.

Alonso, F. \& Chávez, R. (2009). "SIEVP Sistema de Inventario y Evaluación de Viviendas y Pavimentos". Reporte de Investigación autorizado por la Dirección General de Investigación y Posgrado, Universidad Autónoma de Chiapas.

Bellmunt, R., Casanovas, X. y Fernández, M. (2000). "Manual de Diagnosis e Intervención en Estructuras de Hormigón Armado”. Colegio de Apareadores y Arquitectos Técnicos de Barcelona, España.

CFE (2008) "Manual de Diseño de Obras Civiles. Diseño por Sismo", Comisión Federal de Electricidad. Instituto de Investigaciones Eléctricas.

Emmons, P.H. (2005). "Manual Ilustrado de Reparación y Mantenimiento del Concreto". IMCYC, México, 2005.

Escolá, R. (1993). “Construcciones con Defectos, Fallos o Peligros”. Editor Bernando Martin Hernández, Imprenta Berekintza, Bilbao, España

García, F. (2002), "Evaluación de Estructuras de Concreto, Técnicas y Materiales para su Reparación”, IMCYC, México.

Gómez, A. (2008). "Estudio Técnico de las Características y Causas de las Patologías Estructurales en Edificaciones en la Ciudad de Tuxtla Gutiérrez”. Tesis de Grado. Facultad de Ingeniería, Universidad Autónoma de Chiapas.

Muñoz, M. (1991). “Prevención y Soluciones en Patología Estructural de la Edificación”. Mateu Cromo, Madrid, España.

NTC (2004). "Normas Técnicas Complementarias Para diseño y Construcción de Estructuras de Mampostería”, Gaceta Oficial del Distrito Federal, 6 de octubre de 2004, Tomo I No. 103-bis 